\title{
Hexagonal structure of phase III of solid hydrogen
}

\author{
Bartomeu Monserrat,,${ }^{1,2, *}$ Richard J. Needs, ${ }^{2}$ Eugene Gregoryanz, ${ }^{3,4}$ and Chris J. Pickard ${ }^{5,6}$ \\ ${ }^{1}$ Department of Physics and Astronomy, Rutgers University, Piscataway, New Jersey 08854-8019, USA \\ ${ }^{2}$ TCM Group, Cavendish Laboratory, University of Cambridge, \\ J. J. Thomson Avenue, Cambridge CB3 OHE, United Kingdom \\ ${ }^{3}$ School of Physics and Centre for Science at Extreme Conditions, \\ University of Edinburgh, Edinburgh EH9 3JZ, United Kingdom \\ ${ }^{4}$ Key Laboratory of Materials Physics, Institute of Solid State Physics, \\ Chinese Academy of Sciences, Hefei, 230031, China \\ ${ }^{5}$ Department of Materials Science \& Metallurgy, University of Cambridge, \\ 27 Charles Babbage Road, Cambridge CB3 OFS, United Kingdom \\ ${ }^{6}$ Advanced Institute for Materials Research, Tohoku University 2-1-1 Katahira, Aoba, Sendai, 980-8577, Japan
}

(Dated: September 6, 2016)

\begin{abstract}
A hexagonal structure of solid molecular hydrogen with $P 6_{1} 22$ symmetry is calculated to be more stable below about $200 \mathrm{GPa}$ than the monoclinic $C 2 / c$ structure identified previously as the best candidate for phase III. We find that the effects of nuclear quantum and thermal vibrations play a central role in the stabilization of $P 6_{1} 22$. The $P 6_{1} 22$ and $C 2 / c$ structures are very similar and their Raman and infra-red data are in good agreement with experiment. However, our calculations show that the hexagonal $P 6_{1} 22$ structure provides better agreement with the available x-ray diffraction data than the $C 2 / c$ structure at pressures below about $200 \mathrm{GPa}$. We suggest that two phase-III-like structures may be formed at high pressures, hexagonal $P 6_{1} 22$ below about $200 \mathrm{GPa}$ and monoclinic $C 2 / c$ at higher pressures.
\end{abstract}

\section{INTRODUCTION}

Experimental and theoretical studies of hydrogen at high pressures have progressed rapidly in recent years. On the experimental front, improvements in diamond anvil cell techniques have enabled the exploration of static pressures above $300 \mathrm{GPa}$ in hydrogen, ${ }^{1-3}$ and even higher pressures in other materials. ${ }^{4}$ The solid molecular crystalline phases I, II, III, and IV $/ \mathrm{IV}^{\prime}$ of hydrogen have been extensively studied experimentally. However, only the structure of the low temperature and pressure phase I is known with precision, and it is found to be a quantum solid consisting of molecules with angular momentum $L=0$ in a mixture of ortho and para states and arranged on a hexagonal close packed lattice. ${ }^{1-3}$ At low temperatures an pressures above about $100 \mathrm{GPa}$ (25 GPa for deuterium), hydrogen enters phase II in which the molecular rotations are restricted. ${ }^{5}$ The detailed structure of phase II is unknown, although infra-red (IR) and Raman vibrational data, ${ }^{6,7}$ and $\mathrm{x}$-ray and neutron diffraction data ${ }^{8-11}$ impose constraints on it. At about $160 \mathrm{GPa}$, phase II transforms into the ordered molecular phase III. ${ }^{12}$ Phase III has a single strong IR active vibron peak and a much larger IR activity than phase II. ${ }^{13}$ The phases IV and $\mathrm{IV}^{\prime}$, which are similar to eachother, become stable at high pressures and temperatures above about $300 \mathrm{~K} \cdot{ }^{14-16}$ They exhibit a high-frequency vibron peak that is weakly dependent on pressure and a strong Raman vibron peak at lower frequencies which softens rapidly with applied pressure. More recently, a new phase $\mathrm{V}$ of hydrogen has been observed in Raman experiments around room temperature reaching pressures of $388 \mathrm{GPa},{ }^{17}$ but the structure of this new phase also remains unknown.

On the theoretical front, high-pressure structures of hydrogen have been investigated extensively using $a b$ initio molecular dynamics, ${ }^{18-22}$ path-integral molecular dynamics, ${ }^{23,24}$ quantum Monte Carlo methods, ${ }^{3,22,25-30}$, first-principles density functional theory (DFT) methods, ${ }^{31-35}$ and many-body methods. ${ }^{26,36}$ The recent widespread adoption of DFT structure searching techniques has led to the discovery of high-pressure hydrogen structures that are consistent with many of the experimental observations. Using the $a b$ initio random structure searching (AIRSS) method, we found a hydrogen structure of $P 2_{1} / c$ symmetry that is a plausible model for phase II. ${ }^{37}$ We also discovered a monoclinic structure of $C 2 / c$ symmetry and 24 atoms per primitive cell (henceforth called $C 2 / c-24$ ), that provides a good match to the experimental vibrational data for phase III, and is the lowest-enthalpy phase found over the pressure range in which phase III is observed, of 160 to above $300 \mathrm{GPa} .{ }^{32}$ Energetically competitive "mixed structures" of $C 2, P b c n$ and Ibam symmetries were also found $^{32}$ that consist of alternate layers of strongly and weakly bonded molecules, which provide simple models for phases IV $/ \mathrm{IV}^{\prime}$. We have developed improved models for these phases, in particular the $P c$ structure with 48 atoms per primitive unit cell, ${ }^{33,34}$ and also a slightly better structure with 96 atoms per cell (see Supplemental Material of Ref. 33). Structure searching methods have found widespread application beyond hydrogen, discovering many new structures that were subsequently synthesized. For example, AIRSS has been used to determine structures of silane, ${ }^{38}$ aluminum hydride, ${ }^{39}$ ammonia, ${ }^{40,41}$ ammonia hydrates, ${ }^{42}$ and xenon oxides ${ }^{43}$ that were subsequently verified by experiments.

Candidate structures for phases II, ${ }^{37}$ III, ${ }^{32}$ and $\mathrm{IV} / \mathrm{IV}^{\prime 33}$ have been determined by structure searching 
using AIRSS. These searches did not use experimental input, but the resulting structures provide Raman and IR vibrational data in reasonable agreement with experiment. Despite this success, there are still discrepancies between theory and experiment. In particular, there remains an outstanding question about the structure of phase III. Although the vibrational signatures of the monoclinic $C 2 / c-24$ structure agree well with the experimental data for phase III, there is an inconsistency between the experimental x-ray diffraction data for phase III, reported in Ref. 11, and the simulated x-ray data for $C 2 / c-24$. The experimental x-ray data is consistent with a hexagonal space group, but not with the monoclinic space group of $C 2 / c-24 .{ }^{44}$

In this work we investigate this discrepancy using DFT methods. ${ }^{45}$ We find a new hexagonal structure of high pressure hydrogen of $P 6_{1} 22$ symmetry, that is calculated to be more stable than the $C 2 / c-24$ structure below pressures of about $200 \mathrm{GPa}$, once the effects of quantum and thermal motion are incorporated. The Raman and IR spectra of $P 6_{1} 22$ are in good agreement with those observed experimentally for phase III, and the hexagonal symmetry leads to the best agreement of any known candidate structure with the x-ray diffraction data available. We propose $P 6_{1} 22$ as a candidate structure for phase III of solid hydrogen.

The rest of the paper is organized as follows. In Sec. II we describe the structure searches and in Sec. III we calculate the relative free energies of the most competitive candidate structures. We then characterize the new $P 6{ }_{1} 22$ structure in Sec. IV, and propose it as the candidate structure of phase III of solid hydrogen in Sec. V. We draw our conclusions in Sec. VI.

\section{STRUCTURE SEARCHES}

We used AIRSS to search for low enthalpy static-lattice structures of solid hydrogen at high pressures. In contrast to previous searches, we focused on structures containing a number of atoms or molecules equal to a highly composite number. Highly composite numbers are positive integers that have more divisors than any smaller positive integer, and searches over structures containing a highly composite number of atoms or molecules explore structures containing several different numbers of formula units during each search. For each structure, a physically reasonable volume and set of atomic positions were selected at random. Although some searches were performed without symmetry constraints, for most searches we imposed common space group symmetries of molecular crystals, and in particular space groups $P 2_{1} / c$, $P 2_{1} 2_{1} 2_{1}, P c a 2_{1}, P n a 2_{1}$, and $C 2 / c$. We constrained the minimum initial atomic separations using data from preliminary short AIRSS runs, with different minimum separations at each pressure. This helps to space out the atoms appropriately while retaining a high degree of randomness. The structures were then relaxed until the

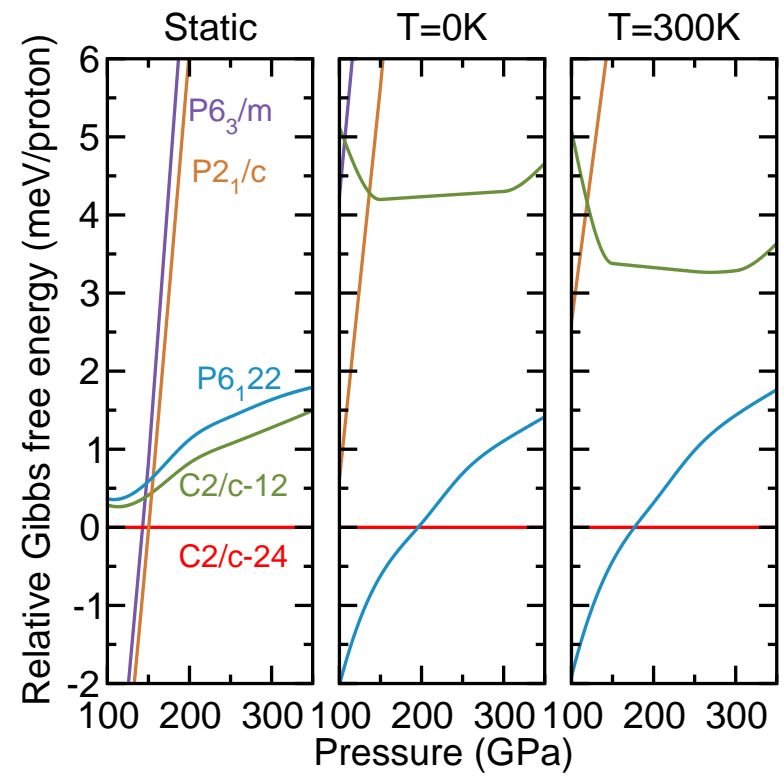

FIG. 1. Relative stability of $P 6_{1} 22$ (blue), $C 2 / c-12$ (green), $P 2_{1} / c$ (orange), and $P 6_{3} / m$ (violet) with respect to $C 2 / c-24$ (red) at the static lattice level, $T=0 \mathrm{~K}$, and $T=300 \mathrm{~K}$.

forces on the atoms were small and the pressure took the required value. This procedure was repeated many times, and a total of 85,424 structures were generated.

The searches were performed using the CASTEP ${ }^{46}$ DFT plane-wave pseudopotential code with "on the fly" ultrasoft pseudopotentials ${ }^{47}$ and the BLYP density functional, ${ }^{48,49}$ which has been shown to provide a good description of molecular hydrogen at high pressures. ${ }^{50}$ We employed an energy cut-off of $230 \mathrm{eV}$, and k-point grids of spacings $2 \pi \times 0.07 \AA^{-1}$ and $2 \pi \times 0.05 \AA^{-1}$.

AIRSS found a previously unknown hydrogen structure of hexagonal $P 6_{1} 22$ symmetry which is energetically competitive with monoclinic $C 2 / c-24$. A primitive unit cell of $P 6_{1} 22$ contains 36 atoms, which is a highly composite number. It appears that this system size had not been explored previously in hydrogen.

\section{FREE ENERGY CALCULATIONS}

We next evaluate the relative enthalpies and free energies of the most competitive structures of high pressure hydrogen in the pressure range 100-350 GPa, as shown in Fig. 1. We include the best known candidate structures for phase II, of $P 2_{1} / c$ and $P 6_{3} / m$ symmetry, the best known candidates for phase III, the $C 2 / c$ - 24 structure, and a 12 atoms variant, referred to as $C 2 / c-12$, as well as the newly discovered $P 6_{1} 22$ structure.

For both static lattice and vibrational energy calculations, we used an energy cut-off of $1000 \mathrm{eV}$ and k-point grids of spacing $2 \pi \times 0.025 \AA^{-1}$. These parameters provide energy differences between frozen-phonon structures that are converged to better than $10^{-4} \mathrm{eV} /$ atom, forces 
to better than $10^{-4} \mathrm{eV} / \AA$, and stresses to better than $10^{-3} \mathrm{GPa}$.

The low mass of hydrogen leads to large vibrational energies and amplitudes and to significant anharmonic nuclear motion, which must be accounted for if accurate energies are to be calculated. We evaluated the free energies using the method proposed in Ref. 51. The low-energy part of the Born-Oppenheimer energy surface was mapped well beyond the harmonic region in a finite-displacement approach. We took advantage of the recently introduced nondiagonal supercells method ${ }^{52}$ to reach unprecedented levels of convergence with respect to the size of the simulation cell. As an example, the results reported here for the $P 6_{1} 22$ structure were obtained using nondiagonal supercells containing a maximum of 72 atoms, but these results are the same as those that would be obtained using the standard supercell approach and a supercell containing 288 atoms. Tests with larger nondiagonal supercells containing a maximum of 108 atoms (equivalent to standard supercells containing 972 atoms) show that the final vibrational energies are converged to better than $0.2 \mathrm{meV} /$ proton. After construction of the anharmonic potential, the resulting Schrödinger equation was solved using a vibrational self-consistent-field approach, in which the vibrational wave function was represented in a basis of simple-harmonic-oscillator functions for each degree of freedom, and converged results were achieved by including up to 50 basis functions per mode.

The relative enthalpies and free energies reported in Fig. 1 correspond to static lattices, $T=0 \mathrm{~K}$, and $T=300 \mathrm{~K}$. At $300 \mathrm{~K}, P 6_{1} 22$ is thermodynamically stable at pressures below about $180 \mathrm{GPa}$ when the vibrational energy is included. The energy difference between $C 2 / c-24$ and $P 6_{1} 22$ is small, but it is clear that the new $P 6_{1} 22$ structure is energetically competitive in the pressure range where phase III is observed experimentally. The structural similarities between $P 6_{1} 22$ and $C 2 / c-24$ suggest that errors in the total free energies arising, for example, from the choice of exchange-correlation functional, should largely cancel when evaluating their relative free energies. The $C 2 / c-12$ structure has a higher static lattice energy than $C 2 / c-24$, and the inclusion of quantum and thermal vibrations destabilizes it further. This demonstrates the importance of the stacking of layers in determining the relative stability of these otherwise very similar structures. The candidate phase II structures are significantly destabilized by the inclusion of quantum nuclear motion, but it has recently been shown that a quantum Monte Carlo description of the electronic energy is necessary to accurately describe the relative energy of these structures compared to $C 2 / c-24 .{ }^{29}$ Finally, we note that even at $300 \mathrm{~K}$, the vibrational energy is dominated by the quantum zero-point motion.

We have also calculated the relative Gibbs free energy of $P 6_{1} 22$ with respect to $C 2 / c-24$ for the heavier deuterium isotope. As atomic vibrations drive the thermodynamic stability of $P 6_{1} 22$ compared to $C 2 / c-24$, the heav-

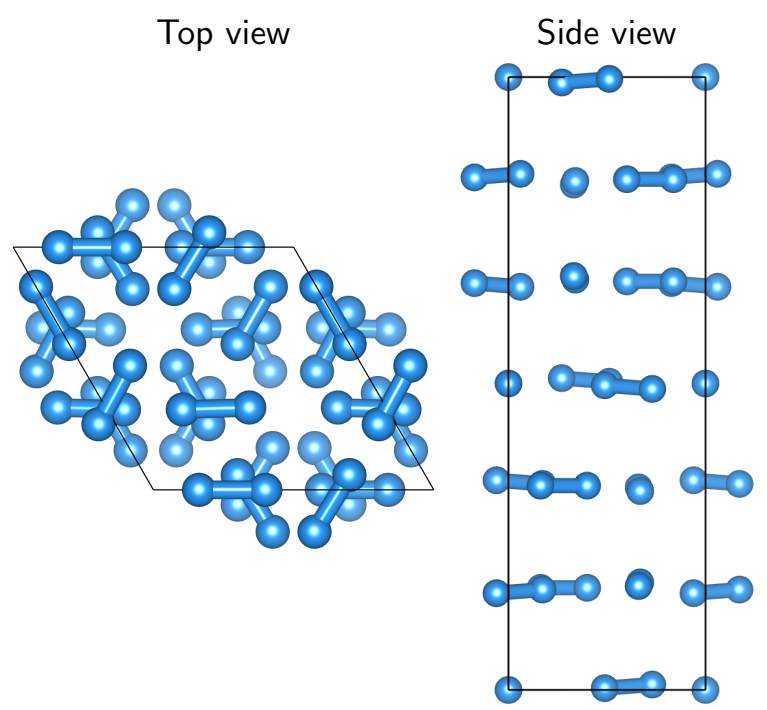

(a) $P 6_{1} 22$.

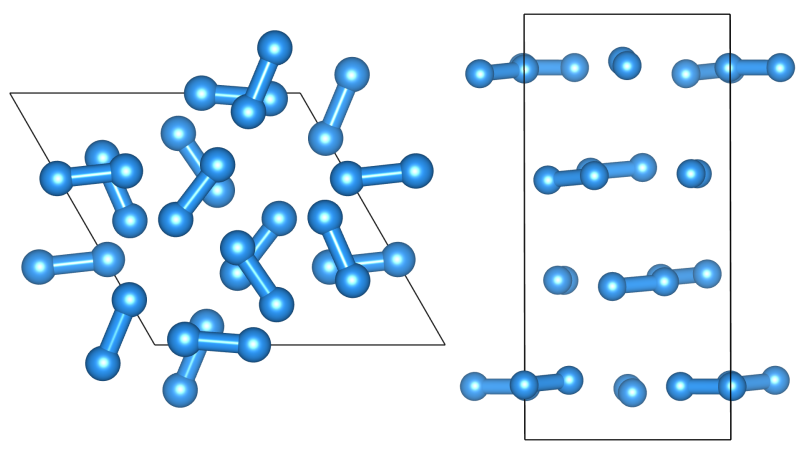

(b) $C 2 / c-24$.

FIG. 2. The $P 6_{1} 22$ and $C 2 / c-24$ layered molecular structures.

ier deuterium compound has a narrower stability range. For example, at $300 \mathrm{~K}$ the transition into the $C 2 / c-24$ structure is predicted to occur at about $160 \mathrm{GPa}$.

\section{PROPERTIES OF THE $P 6_{1} 22$ STRUCTURE}

The data reported in Fig. 1 suggests that $P 6_{1} 22$ is a competitive candidate structure for phase III. Therefore, in this section we characterize the $P 6_{1} 22$ structure, and compare its spectroscopic signatures with experiment and with those of $C 2 / c-24$, which is the best candidate for phase III known at present.

\section{A. Structure}

Both $P 6_{1} 22$ and $C 2 / c-24$ are layered molecular structures, with two extra layers in the hexagonal primitive cell of $P 6_{1} 22$, as shown in Fig. 2. Their structural details at $200 \mathrm{GPa}$ are provided in Table I, which shows 
TABLE I. Static lattice structural details of $C 2 / c-24$ and $P 6_{1} 22$ at $200 \mathrm{GPa}$.

\begin{tabular}{c|ccccccccc}
\hline \hline & $a$ & $b$ & $c$ & $\alpha$ & $\beta$ & $\gamma$ & Volume per proton & BL1 & BL2 \\
\hline$C 2 / c-24$ & $3.025 \AA$ & $3.025 \AA$ & $5.408 \AA$ & $90.1^{\circ}$ & $90.1^{\circ}$ & $119.9^{\circ}$ & $1.787 \AA^{3}$ & $0.719 \AA$ & $0.716 \AA$ \\
$P 6_{1} 22$ & $3.022 \AA$ & $3.022 \AA$ & $8.143 \AA$ & $90.0^{\circ}$ & $90.0^{\circ}$ & $120.0^{\circ}$ & $1.789 \AA^{3}$ & $0.719 \AA$ & $0.715 \AA$ \\
\hline \hline
\end{tabular}

that their primitive cells are similar, differing mainly in the length of the $c$ axis (about $50 \%$ longer in $P 6_{1} 22$ as a consequence of the two extra layers), and in the slight monoclinic distortion in $C 2 / c-24$. Two slightly different molecular bond lengths (BL) appear in these structures, and they differ by less than $0.001 \AA$ between the two structures. The volume per proton of $P 6_{1} 22$ is only $0.1 \%$ larger than that of $C 2 / c-24$. We include a structure file of the $P 6_{1} 22$ structure as Supplemental Material.

\section{B. Bandstructure and phonon dispersion}

In Fig. 3a we show the band structure and density of states of $P 6_{1} 22$ at a pressure of $200 \mathrm{GPa}$. In Fig. 3b we show the corresponding phonon dispersion and associated density of vibrational states. The absence of imaginary frequencies in the phonon dispersion shows that $P 6_{1} 22$ is a dynamically stable structure.

\section{Raman and IR}

The vast majority of experiments on pressurised hydrogen report Raman and/or IR spectra. In Fig. 4 we show the theoretical Raman and IR spectra of $C 2 / c-24$ and $P 6_{1} 22$ at $200 \mathrm{GPa}$. As the $C 2 / c-24$ and $P 6_{1} 22$ structures are almost identical, the frequencies of the active modes are indistinguishable, and agree well with those observed experimentally. ${ }^{13}$ The main difference between the two signals is the stronger IR vibron peak for $P 6_{1} 22$, which is consistent with the observation that in phase III the IR activity is much larger than in phase II. ${ }^{13}$ Overall, the IR and Raman spectra of $C 2 / c-24$ and $P 6_{1} 22$ agree well with the corresponding spectra observed for phase III, and therefore we cannot unambiguously identify the structure of phase III based purely on its vibrational response.

We note that the Raman and IR spectra were obtained using the PBE functional ${ }^{53}$ instead of the BLYP functional. The latter is not implemented in CASTEP within the density functional perturbation theory formalism needed to evaluate these spectra. The main difference between the spectra obtained using PBE and the one that would be obtained using a different functional is the position of the peaks, caused by the slightly different bond lengths predicted by the various functionals.
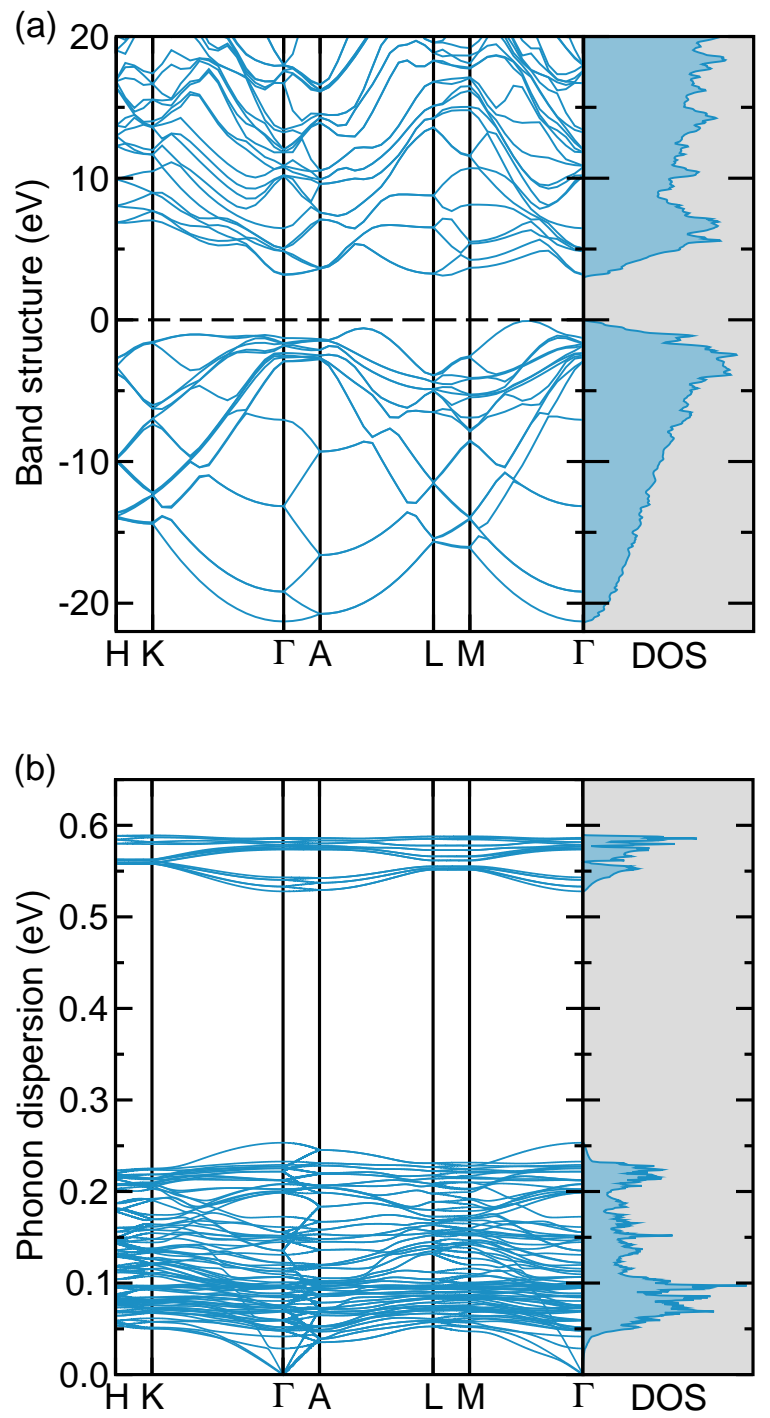

FIG. 3. (a) Band structure along a high-symmetry path (left) and electronic density of states (right) of $P 6{ }_{1} 22$ at $200 \mathrm{GPa}$. The dashed black line represents the Fermi level. (b) Phonon dispersion along a high-symmetry path (left) and vibrational density of states (right) of $P 6_{1} 22$ at $200 \mathrm{GPa}$.

\section{X-ray diffraction}

Hydrogen having the lowest atomic number $\mathrm{Z}$ is a very poor scatterer of x-rays. This, combined with the restrictive access to a diamond anvil cell, makes the structural studies of hydrogen at high pressures notoriously diffi- 


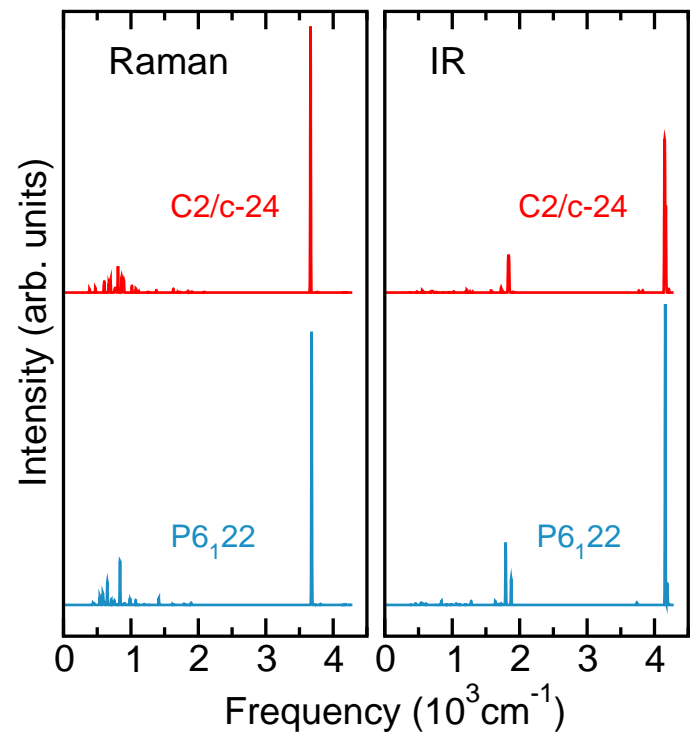

FIG. 4. Raman and IR spectra of $C 2 / c-24$ and $P 6_{1} 22$ at $P=200 \mathrm{GPa}$.

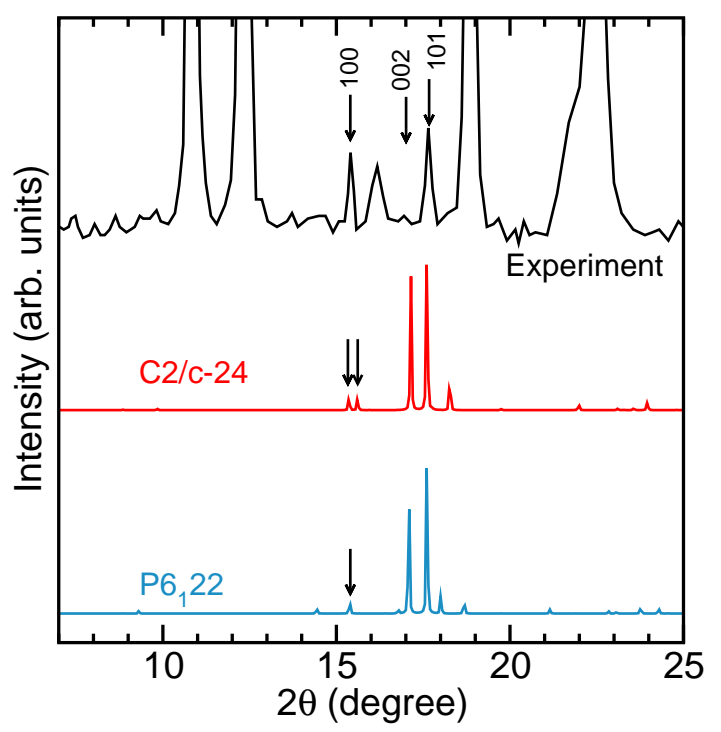

FIG. 5. Experimental x-ray diffraction data for phase III, ${ }^{11}$ and simulated x-ray diffraction data for the $C 2 / c-24$ and $P 6_{1} 22$ structures at $P=174 \mathrm{GPa}$ and wavelength $\lambda=$ $0.4122 \AA$. The black arrows in the experimental data indicate the $\mathrm{H}_{2}$ peaks, while the other peaks correspond to the rhenium metal gasket. The two arrows in the calculated spectrum of $C 2 / c-24$ indicate the double peak at around $15.5^{\circ}$ caused by the monoclinic distortion, and the arrow in the $P 6_{1} 22$ spectrum shows that in this hexagonal structure there is a single peak around $15.5^{\circ}$.

cult: even the structure of phase II, which appears above $25 \mathrm{GPa}$ for deuterium, is still not known. In a remarkable work, Akahama and co-workers recently published x-ray data for phase III of hydrogen up to a pressure of $183 \mathrm{GPa} .{ }^{11}$ Figure 5 shows x-ray diffraction data from Ref. 11, together with our data for $C 2 / c-24$ and $P 6_{1} 22$, simulated using the wavelength $\lambda=0.4122 \AA$ and the lattice parameters corresponding to a pressure of $174 \mathrm{GPa}$. The experimental data for phase III shows two strong reflections at about $15.5^{\circ}$ and $17.7^{\circ}$. These data might show the continuation of the 100 and 101 strong reflections observed in hexagonal phase I. Experimentally, a weaker peak is also observed at about $17.1^{\circ}$ at some pressures, which could be the continuation of the 002 peak of phase I. The 002 peak is not easily observed because the crystallites tend to grow with their $c$-axis perpendicular to the diamond culets, and due to the geometrical constraints of the diamond anvil cell this prevents access to the 001 planes. The available experimental data from Ref. 11 suggests that the x-ray diffraction pattern of a good candidate structure for phase III should (i) exhibit two peaks of similar intensity at $15.5^{\circ}$ and at $17.7^{\circ}$, and (ii) could exhibit a weaker peak at $17.1^{\circ}$.

The x-ray diffraction pattern of $C 2 / c-24$ has a double peak around $15.5^{\circ}$ (see double arrow in Fig. 5), resulting from the monoclinic distortion of the structure, and is inconsistent with the number of peaks observed experimentally. This therefore, rules out $C 2 / c-24$ as a possible structural candidate for phase III below $200 \mathrm{GPa} . P 6_{1} 22$ shows a single peak at $15.5^{\circ}$, which is consistent with its hexagonal symmetry and with experiment. However, the relative peak intensities of $P 6_{1} 22$ are not in good agreement with those observed experimentally. The strongest peaks in $P 6_{1} 22$ are those at $17.1^{\circ}$ and $17.7^{\circ}$, while the peak at $15.5^{\circ}$ is weaker. The intensities of the calculated peaks would match experiment better if the peaks at $15.5^{\circ}$ and $17.1^{\circ}$ were interchanged.

\section{PHASE III OF SOLID HYDROGEN}

The structural and vibrational characteristics of $P 6_{1} 22$, together with the energies reported in Fig. 1, suggest that it is the best candidate for phase III of solid hydrogen of all known structures. It is the first hexagonal candidate for this phase, and its x-ray spectrum exhibits the correct number of peaks at the appropriate locations. The remaining challenge is to explain the discrepancy in the intensities of these peaks between theory and experiment.

Based on the data shown in Fig. 1, we speculate that phase III might in reality be two distinct phases, with the $P 6_{1} 22$ phase being stable below about $200 \mathrm{GPa}$, and $C 2 / c-24$ being stable at higher pressures. The almost identical Raman and IR spectra of these two phases would make it difficult to distinguish between them using spectroscopic techniques, and x-ray data, which might distinguish between the hexagonal and monoclinic symmetries, is only available up to $183 \mathrm{GPa}$, where the hexagonal structure is predicted to be stable. It would be very useful if experimental $\mathrm{x}$-ray data could be collected above 200 GPa. 


\section{CONCLUSIONS}

In conclusion, we have discovered a new candidate structure for phase III of high pressure hydrogen with hexagonal symmetry and space group $P 6_{1} 22$. Our calculations suggest that $P 6_{1} 22$ is the most stable structure at pressures up to about $200 \mathrm{GPa}$, and that its structural and vibrational properties are in better agreement with experiment than any other known candidate structures. Furthermore, the $C 2 / c-24$ structure is predicted to be stable at pressures above $200 \mathrm{GPa}$, which suggests that phase III might be two distinct phases, a hexagonal phase below $200 \mathrm{GPa}$, and a monoclinic phase at higher pressures.

\section{ACKNOWLEDGMENTS}

We thank Nicholas Worth for help with the $\mathrm{x}$ ray diffraction pattern calculations. B.M. acknowledges Robinson College, Cambridge, and the Cambridge Philosophical Society for a Henslow Research Fellowship. C.J.P. and R.J.N. acknowledge financial support from the Engineering and Physical Sciences Research Council (EPSRC) of the UK [EP/K013688/1 and EP/J017639/1]. C.J.P. is also supported by the Royal Society through a Royal Society Wolfson Research Merit award. The calculations were performed on the Darwin Supercomputer of the University of Cambridge High Performance Computing Service facility (http://www.hpc.cam.ac.uk/) and the Archer facility of the UK's national high-performance computing service (for which access was obtained via the UKCP consortium [EP/K014560/1]).
* bm418@cam.ac.uk

1 Isaac F. Silvera, "The solid molecular hydrogens in the condensed phase: Fundamentals and static properties," Rev. Mod. Phys. 52, 393-452 (1980).

2 Ho-kwang Mao and Russell J. Hemley, "Ultrahigh-pressure transitions in solid hydrogen," Rev. Mod. Phys. 66, 671692 (1994).

3 Jeffrey M. McMahon, Miguel A. Morales, Carlo Pierleoni, and David M. Ceperley, "The properties of hydrogen and helium under extreme conditions," Rev. Mod. Phys. 84, 1607-1653 (2012).

4 L. Dubrovinsky, N. Dubrovinskaia, E. Bykova, M. Bykov, V. Prakapenka, C. Prescher, K. Glazyrin, H.-P. Liermann, M. Hanfland, M. Ekholm, Q. Feng, L. V. Pourovskii, M. I. Katsnelson, J. M. Wills, and I. A. Abrikosov, "The most incompressible metal osmium at static pressures above 750 gigapascals," Nature 525, 226 (2015).

5 Isaac F. Silvera and Rinke J. Wijngaarden, "New lowtemperature phase of molecular deuterium at ultrahigh pressure," Phys. Rev. Lett. 47, 39-42 (1981).

6 I. I. Mazin, Russell J. Hemley, A. F. Goncharov, Michael Hanfland, and Ho-kwang Mao, "Quantum and classical orientational ordering in solid hydrogen," Phys. Rev. Lett. 78, 1066-1069 (1997).

7 Alexander F. Goncharov, Eugene Gregoryanz, Russell J. Hemley, and Ho-kwang Mao, "Spectroscopic studies of the vibrational and electronic properties of solid hydrogen to 285 GPa," Proc. Natl. Acad. Sci. 98, 14234-14237 (2001).

8 P. Loubeyre, R. LeToullec, D. Hausermann, M. Hanfland, R. J. Hemley, H. K. Mao, and L. W. Finger, "X-ray diffraction and equation of state of hydrogen at megabar pressures," Nature 383, 702 (1996).

9 H. Kawamura, Y. Akahama, S. Umemoto, K. Takemura, Y. Ohishi, and O. Shimomura, "X-ray powder diffraction from solid deuterium," J. Phys.: Condens. Matter 14, 10407 (2002).

10 Igor Goncharenko and Paul Loubeyre, "Neutron and x-ray diffraction study of the broken symmetry phase transition in solid deuterium," Nature 435, 1206 (2005).
11 Yuichi Akahama, Manabu Nishimura, Haruki Kawamura, Naohisa Hirao, Yasuo Ohishi, and Kenichi Takemura, "Evidence from x-ray diffraction of orientational ordering in phase III of solid hydrogen at pressures up to $183 \mathrm{GPa}$," Phys. Rev. B 82, 060101 (2010).

12 R. J. Hemley and H. K. Mao, "Phase transition in solid molecular hydrogen at ultrahigh pressures," Phys. Rev. Lett. 61, 857-860 (1988).

13 Russell J. Hemley, Zoltan G. Soos, Michael Hanfland, and Ho-kwang Mao, "Charge-transfer states in dense hydrogen," Nature 369, 384 (1994).

14 M. I. Eremets and I. A. Troyan, "Conductive dense hydrogen," Nat. Mater. 10, 927 (2011).

15 Ross T. Howie, Christophe L. Guillaume, Thomas Scheler, Alexander F. Goncharov, and Eugene Gregoryanz, "Mixed molecular and atomic phase of dense hydrogen," Phys. Rev. Lett. 108, 125501 (2012).

16 Ross T. Howie, Thomas Scheler, Christophe L. Guillaume, and Eugene Gregoryanz, "Proton tunneling in phase IV of hydrogen and deuterium," Phys. Rev. B 86, 214104 (2012).

17 Philip Dalladay-Simpson, Ross T. Howie, and Eugene Gregoryanz, "Evidence for a new phase of dense hydrogen above 325 gigapascals," Nature 529, 63 (2016).

18 Jorge Kohanoff, Sandro Scandolo, Guido L. Chiarotti, and Erio Tosatti, "Solid molecular hydrogen: The broken symmetry phase," Phys. Rev. Lett. 78, 2783-2786 (1997).

19 Jorge Kohanoff, Sandro Scandolo, Stefano de Gironcoli, and Erio Tosatti, "Dipole-quadrupole interactions and the nature of phase III of compressed hydrogen," Phys. Rev. Lett. 83, 4097-4100 (1999).

20 Ioan B. Magdău and Graeme J. Ackland, "Identification of high-pressure phases III and IV in hydrogen: Simulating Raman spectra using molecular dynamics," Phys. Rev. B 87, 174110 (2013).

21 Ranber Singh, Sam Azadi, and Thomas D. Kühne, "Anharmonicity and finite-temperature effects on the structure, stability, and vibrational spectrum of phase III of solid molecular hydrogen," Phys. Rev. B 90, 014110 (2014). 
22 Ji Chen, Xinguo Ren, Xin-Zheng Li, Dario Alfè, and Enge Wang, "On the room-temperature phase diagram of high pressure hydrogen: An ab initio molecular dynamics perspective and a diffusion Monte Carlo study," J. Chem. Phys. 141, 024501 (2014).

${ }^{23}$ Hikaru Kitamura, Shinji Tsuneyuki, Tadashi Ogitsu, and Takashi Miyake, "Quantum distribution of protons in solid molecular hydrogen at megabar pressures," Nature 404, 259 (2000).

24 Ji Chen, Xin-Zheng Li, Qianfan Zhang, Matthew I. J. Probert, Chris J. Pickard, Richard J. Needs, Angelos Michaelides, and Enge Wang, "Quantum simulation of low-temperature metallic liquid hydrogen," Nat. Commun. 4, 2064 (2013).

25 D. M. Ceperley and B. J. Alder, "Ground state of solid hydrogen at high pressures," Phys. Rev. B 36, 2092-2106 (1987).

26 Sam Azadi, W. M. C. Foulkes, and Thomas D. Kühne, "Quantum Monte Carlo study of high pressure solid molecular hydrogen," New J. Phys. 15, 113005 (2013).

27 Sam Azadi, Bartomeu Monserrat, W. M. C. Foulkes, and R. J. Needs, "Dissociation of high-pressure solid molecular hydrogen: A quantum Monte Carlo and anharmonic vibrational study," Phys. Rev. Lett. 112, 165501 (2014).

28 Jeremy McMinis, Raymond C. Clay, Donghwa Lee, and Miguel A. Morales, "Molecular to atomic phase transition in hydrogen under high pressure," Phys. Rev. Lett. 114, 105305 (2015).

29 N. D. Drummond, B. Monserrat, J. H. Lloyd-Williams, P. López Ríos, C. J. Pickard, and R. J. Needs, "Quantum Monte Carlo study of the phase diagram of solid molecular hydrogen at extreme pressures," Nat. Commun. 6, 7794 (2015).

30 Sam Azadi, N. D. Drummond, and W. M. C. Foulkes, "Nature of the metallization transition in solid hydrogen," arxiv:1608.00754 (2016).

31 Kurt A. Johnson and N. W. Ashcroft, "Structure and bandgap closure in dense hydrogen," Nature 403, 632 (2000).

32 Chris J. Pickard and Richard J. Needs, "Structure of phase III of solid hydrogen," Nat. Phys. 3, 473-476 (2007).

33 Chris J. Pickard, Miguel Martinez-Canales, and Richard J. Needs, "Density functional theory study of phase IV of solid hydrogen," Phys. Rev. B 85, 214114 (2012).

34 Chris J. Pickard, Miguel Martinez-Canales, and Richard J. Needs, "Erratum: Density functional theory study of phase IV of solid hydrogen [Phys. Rev. B 85, 214114 (2012)]," Phys. Rev. B 86, 059902(E) (2012).

35 S. Azadi and Th. D. Kühne, "Absence of metallization in solid molecular hydrogen," JETP Lett. 95, 449 (2012).

36 Marc Dvorak, Xiao-Jia Chen, and Zhigang Wu, "Quasiparticle energies and excitonic effects in dense solid hydrogen near metallization," Phys. Rev. B 90, 035103 (2014).

37 C. J. Pickard and R. J. Needs, "Structures at high pressure from random searching," Phys. Status Solidi B 246, 536540 (2009).

${ }^{38}$ Chris J. Pickard and R. J. Needs, "High-pressure phases of silane," Phys. Rev. Lett. 97, 045504 (2006).
39 Chris J. Pickard and R. J. Needs, "Metallization of aluminum hydride at high pressures: A first-principles study," Phys. Rev. B 76, 144114 (2007).

40 Chris J. Pickard and R. J. Needs, "Highly compressed ammonia forms an ionic crystal," Nat. Mater. 7, 775 (2008).

41 S. Ninet, F. Datchi, P. Dumas, M. Mezouar, G. Garbarino, A. Mafety, C. J. Pickard, R. J. Needs, and A. M. Saitta, "Experimental and theoretical evidence for an ionic crystal of ammonia at high pressure," Phys. Rev. B 89, 174103 (2014).

42 A. Dominic Fortes, Emmanuelle Suard, Marie-Hélène Lemée-Cailleau, Christopher J. Pickard, and Richard J. Needs, "Crystal structure of ammonia monohydrate phase II," J. Amer. Chem. Soc. 131, 13508-13515 (2009).

43 Agnès Dewaele, Nicholas Worth, Chris J. Pickard, Richard J. Needs, Sakura Pascarelli, Olivier Mathon, Mohamed Mezouar, and Tetsuo Irifune, "Synthesis and stability of xenon oxides $\mathrm{Xe}_{2} \mathrm{O}_{5}$ and $\mathrm{Xe}_{3} \mathrm{O}_{2}$ under pressure," Nat. Chem. 8, 784 (2016).

44 Alexander F. Goncharov, Ross T. Howie, and Eugene Gregoryanz, "Hydrogen at extreme pressures," Low Temp. Phys. 39, 402-408 (2013).

45 R. O. Jones, "Density functional theory: Its origins, rise to prominence, and future," Rev. Mod. Phys. 87, 897-923 (2015).

46 Stewart J. Clark, Matthew D. Segall, Chris J. Pickard, Phil J. Hasnip, Matt I. J. Probert, Keith Refson, and Mike C. Payne, "First principles methods using CASTEP," Z. Kristallogr. 220, 567 (2005).

47 David Vanderbilt, "Soft self-consistent pseudopotentials in a generalized eigenvalue formalism," Phys. Rev. B 41, 7892-7895 (1990).

48 A. D. Becke, "Density-functional exchange-energy approximation with correct asymptotic behavior," Phys. Rev. A 38, 3098-3100 (1988).

49 Chengteh Lee, Weitao Yang, and Robert G. Parr, "Development of the Colle-Salvetti correlation-energy formula into a functional of the electron density," Phys. Rev. B 37, 785-789 (1988).

50 Raymond C. Clay, Jeremy Mcminis, Jeffrey M. McMahon, Carlo Pierleoni, David M. Ceperley, and Miguel A. Morales, "Benchmarking exchange-correlation functionals for hydrogen at high pressures using quantum Monte Carlo," Phys. Rev. B 89, 184106 (2014).

51 Bartomeu Monserrat, N. D. Drummond, and R. J. Needs, "Anharmonic vibrational properties in periodic systems: Energy, electron-phonon coupling, and stress," Phys. Rev. B 87, 144302 (2013).

52 Jonathan H. Lloyd-Williams and Bartomeu Monserrat, "Lattice dynamics and electron-phonon coupling calculations using nondiagonal supercells," Phys. Rev. B 92, 184301 (2015).

53 John P. Perdew, Kieron Burke, and Matthias Ernzerhof, "Generalized gradient approximation made simple," Phys. Rev. Lett. 77, 3865 (1996). 